\title{
Status Epilepticus in Neonates
}

\author{
Arushi Gahlot Saini ${ }^{1}$ Kollencheri Puthenveettil Vinayan² \\ ${ }^{1}$ Pediatric Neurology Unit, Department of Pediatrics, Postgraduate \\ Institute of Medical Education and Research, Chandigarh, India \\ ${ }^{2}$ Division of Pediatric Neurology, Department of Neurology, \\ Amrita Institute of Medical Sciences, Amrita University, Cochin, \\ Kerala, India
}

\begin{abstract}
Address for correspondence Kollencheri Puthenveettil Vinayan Division of Pediatric Neurology, Department of Neurology, Amrita Institute of Medical Sciences, Cochin, 682041, Kerala, India (e-mail: vinayankp@aims.amrita.edu).
\end{abstract}

Int J Epilepsy 2018;5:2-8

\begin{abstract}
Keywords

- neonatal seizures

- neonatal status epilepticus

- status epilepticus

- antiepileptic

- brain injury

Seizures are a reflection of acute brain injury in the neonatal period, and status epilepticus reflects a state of high seizure burden. Neonatal status epilepticus has been reported in 8 to $43 \%$ of newborns with seizures. There is no separate definition for neonatal status epilepticus, and it has been commonly defined as a continuous seizure lasting at least 30 minutes and/or a series of seizures whose total duration exceeds $50 \%$ of a given epoch. The causes of status epilepticus in the neonatal period mirror the causes of neonatal seizures. It is symptomatic, usually associated with hypoxic-ischemic encephalopathy, inherited metabolic disorders, infections, and cerebral hemorrhage. Management guidelines are not separately outlined for neonatal status epilepticus and are commonly derived from the recommendations for neonatal and infantile seizures. The presence of neonatal status epilepticus significantly increases the odds for development of cerebral palsy, global developmental delay, and epilepsy later in life. Further research is needed into the role of current antiepileptic drugs in causing neuronal injury and use of neuroprotective agents during neonatal status epilepticus.
\end{abstract}

\section{Introduction}

Seizures are the most common reflection of an acute brain injury in the neonatal period. The first 4 weeks of life (neonatal period) are at the highest risk for seizures secondary to an underlying predisposition of the immature brain as well as the nature of the inciting injury. Seizures occur in 1 to 3.5 per 1,000 births. $^{1}$ The neonatal seizure continuum ranges from isolated events to recurrent seizures and status epilepticus. Neonatal status epilepticus (NSE) reflects a high seizure burden state and is associated with worse neurologic outcomes compared with recurrent seizures. ${ }^{2}$

NSE is distinct from status epilepticus in children and adults in definition, etiopathogenesis, electroencephalographic (EEG) features, management, and outcomes. It resembles neonatal seizures more than it does status epilepticus at later ages. However, management recommendations are currently based on those for children and adults with some age-specific modifications. We discuss here the unique features of NSE and discuss the current recommendations for its diagnosis and management.

\section{The Unique Neurobiology of a Neonatal Brain}

Neonatal seizure and status epilepticus are unique as the neonatal brain differs in both structure and physiologic functioning from the brains of children and adults. The neonatal brain has a distinct predilection for neuronal excitation and subsequent seizures. Several probable neurobiological mechanisms have been put forward to explain this increased susceptibility:

- There is an overexpression of glutamate (the major excitatory neurotransmitter of the central nervous system) as well as altered expression of its $N$-methyl-Daspartate (NMDA) (overexpression of NR2B subunits) and $\alpha$-amino-3-hydroxy-5-methyl-4-isoxazolepropionic acid (AMPA) receptors (lack of GluR2 subunit).,4,

- There is reduction in $\gamma$-aminobutyric acid (GABA) (the major inhibitory neurotransmitter in the central nervous system), its receptor expression, and alteration in its distribution (overexpression of $\alpha 4$ and $\alpha 2$ subunits compared with $\alpha 1$ ) leading to benzodiazepine resistance. ${ }^{5}$
DOI https://doi.org/

10.1055/s-0038-1667213.

ISSN 2213-6320.
License terms

()(1) $\Theta \circledast$ 
- There is underexpression of $\mathrm{KCC} 2$ (pumps $\mathrm{Cl}^{-}$ions out) and overexpression of NKCC1 (pumps $\mathrm{Cl}^{-}$in) channels on the immature neonatal neurons resulting in a higher intracellular $\mathrm{Cl}^{-}$ion concentrations. GABA-agonist medications such as phenobarbitone and benzodiazepines (meant to cause neuronal hyperpolarization in a mature brain) thus result in paradoxical depolarization in the immature neurons due to the net efflux of $\mathrm{Cl}^{-}$ions. ${ }^{6}$

- This $\mathrm{Cl}^{-}$ion gradient matures in a caudo-rostral direction during development. This maturational gradient may lead to inhibition of motor neurons in the spinal cord and brainstem while depolarizing the cortical neurons with the use of GABA-agonists, resulting in electromechanical dissociation with phenobarbitone and midazolam use. ${ }^{7}$

- In the neonatal brain, although the basic organization of the cerebral cortex remains the same as in older children, the dendritic arborization, connections, and synaptic stabilizations are incomplete and the myelination is immature. This prevents rapid, well-organized spread of epileptic discharges in the brain and absence of the classic type of generalized, tonic-clonic status epilepticus in neonates. Additionally, the electroclinical dissociation and presence of subtle nonobtrusive signs such as autonomic variations, apnea, and oculomotor or oral-buccal-lingual movements in neonatal seizures suggest the possibility of a subcortical generator rather the overlying cerebral cortex as in older children and adults. ${ }^{1}$

- Based on serial electrographic maturational changes during status epilepticus during the first 6 months of life, it has been suggested that infants older than 2 months start exhibiting electrographic features similar to those of status epilepticus seen in older children such as presence of prolonged seizures or partial seizures with one focus and secondary generalization. ${ }^{8}$ However, the electrographic features of NSE in infants younger than 2 months are usually distinct as described above.

\section{Definitions}

The conventional definition describes status epilepticus in children and adults as "any continuous seizure activity lasting $\geq 30$ minutes or $\geq 2$ seizures without regaining baseline mental status in between the events." An operational definition of status epilepticus proposes "any continuous clinical seizure activity lasting for $\geq 5$ minutes or $\geq 2$ seizures without regaining baseline mental status in between." ${ }^{10}$ Recently, the International League Against Epilepsy (ILAE) has revised the definition of status epilepticus as "a condition resulting either from failure of the mechanisms responsible for seizure termination or from the initiation of mechanisms, which lead to abnormally, prolonged seizures (after time point $t$ ), and long-term consequences (after time-point t2), including neuronal death, neuronal injury, and alteration of neuronal networks, depending on the type and duration of seizures."11 Duration longer than 5 minutes has been considered "prolonged" and longer than 30 minutes has been associated with sequelae for generalized tonic clonic seizures. ${ }^{12}$
The latest ILAE report suggests classifying neonatal seizures according to the same descriptors as other seizures, rather than a separate entity..$^{13}$ NSE has not been as clearly defined although the 5-minute duration seems more logical as majority of neonatal seizures do not last $>2$ to 3 minutes. ${ }^{14,15}$ It has been variably defined as a "continuous seizure activity lasting $>30$ minutes and/or a series of seizures whose total duration exceeds 50\% of a given epoch."2,16 Given the worse outcomes associated with NSE and the need for prompt treatment, a clinical definition to treat "all frequent or prolonged seizures $\geq 5$ minutes in duration as status epilepticus" has also been used. ${ }^{2,17}$ This variability in defining NSE has led to inconsistent estimates (8-43\%) of the burden of NSE in newborns with seizures..$^{18-20}$ The challenges in defining neonatal seizures and status epilepticus are ${ }^{21}$ :

- The seizure semiology, clinical and EEG characteristics in neonates are different compared with older children. Most seizures in the neonates are either subtle, multifocal, migratory, or rarely fully generalized whereas majority of the current literature of status epilepticus is based on well-organized, generalized tonic-clonic status.

- As the average duration of neonatal seizures ranges from 1 to 5 minutes, and solitary prolonged seizures are rare; hence, a 30-minute definition would underdiagnose NSE. ${ }^{22}$

- Neonates with seizures and status are commonly critically ill and encephalopathic, thus making the recognition of baseline mental status or a return to it difficult.

- Nearly 80 to $90 \%$ of electrographic seizures lack a clinical correlate and would not be identified without continuous EEG. ${ }^{23}$ Further, anticonvulsant administration may terminate clinically evident seizures whereas electrographic-only seizures may persist resulting in electroclinical uncoupling or dissociation.

- Defining NSE as any electrographic recording with seizure activity noted over a certain (most commonly $>50 \%$ ) proportion of the length of the recording time ${ }^{24}$ would not be useful and difficult to apply in resource-limited settings where routine continuous EEG monitoring is not available and interictal recording times vary. Also, there is a clear lack of evidence to support any particular threshold to designate a high seizure burden as SE. ${ }^{25}$

\section{Etiology}

In general, the causes of NSE mirror the causes of neonatal seizures that are most often symptomatic markers of brain injury. ${ }^{26}$ The most common cause of recurrent seizures in the newborn is hypoxic ischemic encephalopathy $(50-60 \%){ }^{20}$ These seizures are noted within the first 12 to 24 hours of life, associated with encephalopathy, and are subtle and multifocal in nature. The next most common cause is vascular, either arterial or venous strokes, resulting in focal seizures after 24 hours of age and associated with minimal baseline encephalopathy. An important cause of NSE is intracranial hemorrhage, isolated or more commonly associated with hypoxic ischemic encephalopathy or stroke. Intraventricular hemorrhage, in particular, may cause subtle seizures in the preterm neonate. ${ }^{27}$ Other causes include central nervous 
system infections, congenital malformations, acute or inherited metabolic disorders, local anesthetic intoxication, drug withdrawal, and hereditary epilepsies. ${ }^{21}$ Although acute transient metabolic disorders such as hypoglycemia, hypocalcemia, hypomagnesemia, or hypophosphatemia cause recurrent seizures more than NSE, these must be recognized early as specific therapy is warranted in these cases. Neonatal seizures need to be differentiated from neonatal-onset inherited epilepsies. Inborn errors of metabolism should be suspected in the absence of an acute insult or structural cause when seizures are accompanied by encephalopathy and when seizures are refractory to the standard anticonvulsants ( - Table $\mathbf{1}$ ).

\section{Approach to Neonatal Status Epilepticus}

The diagnosis of neonatal seizures is largely clinical in routine practice, and a detailed approach has been described elsewhere. ${ }^{28}$ Nevertheless, in a neonate with suspected or confirmed status epilepticus, five questions need to be asked ${ }^{29}$ :

- Are the clinical events seizures or mimics? The answer to this question often requires an EEG correlate of the ictal activity. ${ }^{29}$ Whereas focal clonic and tonic seizures have a good EEG correlate, subtle and myoclonic seizures correlate poorly with the EEG. Epileptic movements in the neonates and infants include generalized myoclonic jerks, myoclonic jerks of the diaphragm leading to frequent hiccups (glycine encephalopathy), clonic limb jerking, tongue thrusting, focal tonic posturing when associated with autonomic features, and tonic eye deviation and epileptic spasms. ${ }^{30}$ Movements that are less likely to be epileptic without further investigation include cycling, pedaling or thrashing of limbs, side-to-side movements of the head, sucking, lip puckering, grimacing, tongue protrusion, blinking without tonic eye deviation, roving eye movements, nystagmus, and generalized tonic stiffening. ${ }^{30}$
Common nonepileptic movements include recurrent tremors or jitteriness, clonus, myoclonus only seen in sleep, dystonia, and startle with or without tonic stiffening (hereditary hyperexplexia). ${ }^{31}$

- Is there a clinical or investigative evidence for a preexisting neurologic condition in the neonate such as malformations, genetic disorders, or remote structural injuries?..$^{29}$ Clinical clues to an underlying predisposition include presence of microcephaly, dysmorphic features, or genetic or neuroimaging abnormality.

- Are seizures provoked or unprovoked? Majority of neonatal seizures are acute symptomatic seizures secondary to a hypoxic, ischemic, infective, vascular or metabolic disturbance, and need correction of underlying abnormality besides antiepileptic drugs. Unprovoked seizures associated with genetically determined early-onset epileptic encephalopathies need specific investigations and therapy.

- Are the seizures themselves associated with an increased risk of brain injury and subsequent poorer neurodevelopmental outcome? ${ }^{29}$ NSE has been associated with a lower full-scale intelligent quotient and increased risk of subsequent epilepsy. ${ }^{32}$

- Does the antiseizure treatment outweigh the risks of ongoing seizures? The answer to this question is particularly difficult as the short- and long-term side effects of antiepileptic drugs are not known in NSE.

The clinical presentations of recurrent neonatal seizures and status epilepticus can be variable and many are subclinical. Hence, clinical identification alone may be unreliable and insufficient. Investigations in NSE are limited, and seizure control takes a priority as ongoing seizures have been shown to cause long-term neuronal loss and cognitive impact in animal studies. Nevertheless, appropriate blood and cerebrospinal fluid investigations to rule out underlying metabolic abnormalities or infection and neuroimaging are essential

Table 1 Common causes for recurrent neonatal seizures and neonatal status epilepticus

\begin{tabular}{|l|}
\hline Hypoxic-ischemic encephalopathy \\
\hline Intracerebral hemorrhage \\
\hline Ischemic stroke \\
\hline Cerebral venous thrombosis \\
\hline Central nervous system infections \\
\hline $\begin{array}{l}\text { Acquired metabolic insults } \\
\text { Hypoglycemia, hypocalcemia, hypomagnesemia, hypophosphatemia, hyperbilirubinemia }\end{array}$ \\
\hline Narcotic withdrawal \\
\hline Trauma \\
\hline Brain malformation \\
\hline $\begin{array}{l}\text { Neonatal epilepsies (genetic) } \\
\text { Benign familial neonatal epilepsy, early myoclonic epileptic encephalopathy }\end{array}$ \\
\hline $\begin{array}{l}\text { Inborn errors of metabolism (genetic and in need of specific dietary or cofactor therapy) } \\
\text { Pyridoxine dependent epilepsy, folinic acid responsive seizures, nonketotic hyperglycinemia, sulfite oxidase deficiency, } \\
\text { molybdenum cofactor deficiency, holocarboxylase, biotinidase deficiency, organic acidurias, Menkes disease, peroxisomal } \\
\text { disorders, hereditary fructose intolerance, fructose 1,6-diphosphate deficiency, urea cycle defects }\end{array}$ \\
\hline
\end{tabular}


investigations. Specifically, a lumbar puncture analysis also aids in the diagnosis in case of glucose transporter defects (with a cerebrospinal fluid-to-plasma glucose ratio < 0.35 ), mitochondrial cytopathies (elevated lactate), and glycine encephalopathy (with a cerebrospinal fluid-to-plasma glycine ratio > 0.08). Correct identification, evolution, and prognostication are enhanced by the use of EEG or amplitude-integrated EEG (aEEG) monitoring of high-risk infants. Although continuous EEG monitoring and intensive care is recommended in all cases of status epilepticus, the same may not be feasible in resource-limited emergency settings. ${ }^{33,34}$ The American Clinical Neurophysiology Society's guideline on continuous EEG monitoring in neonates states that "conventional video-EEG monitoring is the gold standard for neonatal seizure detection and quantification and should be used whenever available for seizure detection and differential diagnosis of abnormal appearing, paroxysmal clinical events. It is the ideal tool to measure the exact number and duration of seizures, their site(s) of onset and spatial patterns of migration." ${ }^{35}$ However, in resource-limited settings, at least a routine EEG is warranted in all cases of recurrent neonatal seizures, in cases of persisting neonatal encephalopathy with or without anticonvulsant or neuromuscular blockade to rule out epileptic encephalopathies, subclinical or nonconvulsive seizures. The neonatal interictal EEG recording should last minimum of 1 hour to capture all stages of sleep. A reduced-montage EEG recording, aEEG, is gaining popularity in many neonatal intensive care units due to its simplified display of one or two channels of time-compressed, processed EEG signal on a semilogarithmic scale. The interpretation can be done by the treating physician/neurologist at the bedside by pattern identification and is enhanced by concurrent display of the raw signal or multichannel EEG. Although the aEEG background patterns have been shown to have predictive value for neonates with encephalopathy, including hypoxic ischemic encephalopathy, its use for detection of seizures is controversial due to its very low sensitivity.

As in older children and adults, NSE is a life-threatening condition requiring rapid treatment and prevention of neuronal death as well as secondary injury. ${ }^{36}$ Time is the key in the management of status epilepticus because as many as $43 \%$ of neonatal seizures may progress into status epilepticus. Seizures become more difficult to treat the longer they last, and a higher seizure burden portends worse outcomes. ${ }^{37}$ The management of NSE begins with maintenance of airway, breathing and circulation; assessment of the etiological cause; and simultaneous use of anticonvulsant drugs, especially if the baby is sick or encephalopathic (-Fig. 1). In the majority of NSEs, the underlying cause of symptomatic seizures such as hypoxic-ischemic encephalopathy, stroke or hemorrhage, and infections need to be dealt with appropriate measures simultaneously. A trial of intravenous glucose and calcium may be considered in neonates with seizure at the onset and simultaneous samples to be collected for evaluation of hypoglycemia, hypocalcemia, and hypomagnesemia, especially in absence of history of hypoxic-ischemic injury. Evidence-based guidelines for optimal antiepileptic management of NSE are currently not available. The current anticonvulsant drugs are effective in nearly $50 \%$ patients as a single agent and in 60 to $70 \%$ patients with multiple agents. ${ }^{38}$ Phenobarbitone remains the drug of choice as first-line agent (dose $15-20 \mathrm{mg} / \mathrm{kg}$ loading dose by intravenous route that may be repeated and is followed by maintenance dosages of $3-5 \mathrm{mg} / \mathrm{kg} /$ day) to treat recurrent neonatal seizures and status epilepticus although concerns have been raised over the risk of neuronal apoptosis with increasing serum concentrations of the drug. ${ }^{39}$ The second-line agent is either phenytoin or fosphenytoin (dose $15-20 \mathrm{mg} / \mathrm{kg}$ loading dose by intravenous route followed by maintenance dosages of $5-8 \mathrm{mg} / \mathrm{kg} /$ day). Benzodiazepines are commonly used next. Intravenous lorazepam (loading dose of $0.05-0.10 \mathrm{mg} / \mathrm{kg}$ in 5 minutes) is used intermittently with a rapid response, half-life of 40 hours, and minimal side effects in neonates. Alternatively, midazolam infusion (incremental dose of $0.1-0.5 \mathrm{mg} / \mathrm{kg} / \mathrm{h}$, maximum up to $1 \mathrm{mg} / \mathrm{kg} / \mathrm{h}$ ) is used along with intensive monitoring for respiratory depression. ${ }^{40}$ By this time, it is prudent to administer empiric therapy and/or exclude treatable metabolic disorders such as pyridoxine dependent epilepsy (dose of $100 \mathrm{mg}$ intravenous pyridoxine during EEG monitoring followed by $30 \mathrm{mg} / \mathrm{kg} /$ day divided into two to three doses by oral or intravenous route over 3 consecutive days), folinic-acid responsive seizures ( $2.5 \mathrm{mg}$ intravenous or oral folinic acid in a dose of $3-5 \mathrm{mg} / \mathrm{kg} /$ day in two to three divided doses over 3 days), and biotinidase deficiency (oral biotin in a dose of $5-10 \mathrm{mg} /$ day). In persisting seizures, a trial of pyridoxal-5'-phosphate (dose of $30-50 \mathrm{mg} / \mathrm{kg} /$ day in three to four divided doses over 3 days) is warranted. ${ }^{41}$ Also, continuous EEG monitoring should be considered at this point as the subclinical seizures may persist. Newer antiepileptic drugs such as levetiracetam (loading dose of $20-30 \mathrm{mg} / \mathrm{kg}$ followed by initial maintenance dose of $5-10 \mathrm{mg} / \mathrm{kg} /$ day, which may be increased if required), lidocaine, and oral topiramate are the next line agents depending on the feasibility and availability of the drugs. Although limited safety and efficacy data are available on the use of levetiracetam in neonates, its use is increasing due to the easy availability of intravenous formulation and favorable side effect profile among older children and adults. ${ }^{42}$ The drug has received approval of the Food and Drug Administration (FDA) for use in infants 1 month of age and older in 2011. Seizure control within 1 hour has been achieved in up to $86 \%$ neonates treated with intravenous levetiracetam..$^{43}$ Additionally, levetiracetam is a good choice in neonates with cardiac or liver dysfunction or acute brain injury. Levetiracetam does not enhance neuronal apoptosis in the developing brain and might have neuroprotective effects. ${ }^{44}$ Drugs rarely used for NSE include valproate, paraldehyde, vigabatrin, and carbamazepine. ${ }^{45}$ Therapeutic hypothermia has emerged as a useful neuroprotective strategy for newborns with mild to moderate hypoxic ischemic encephalopathy and seizures, which show a significantly reduced seizure burden ${ }^{46}$ The study on the use of bumetanide as an adjuvant to phenobarbital for the treatment of neonatal seizures posthypoxic ischemic encephalopathy (NEMO [treatment of NEonatal seizure using Medication Off-patent] trial) demonstrated serious side effects including hearing loss and 


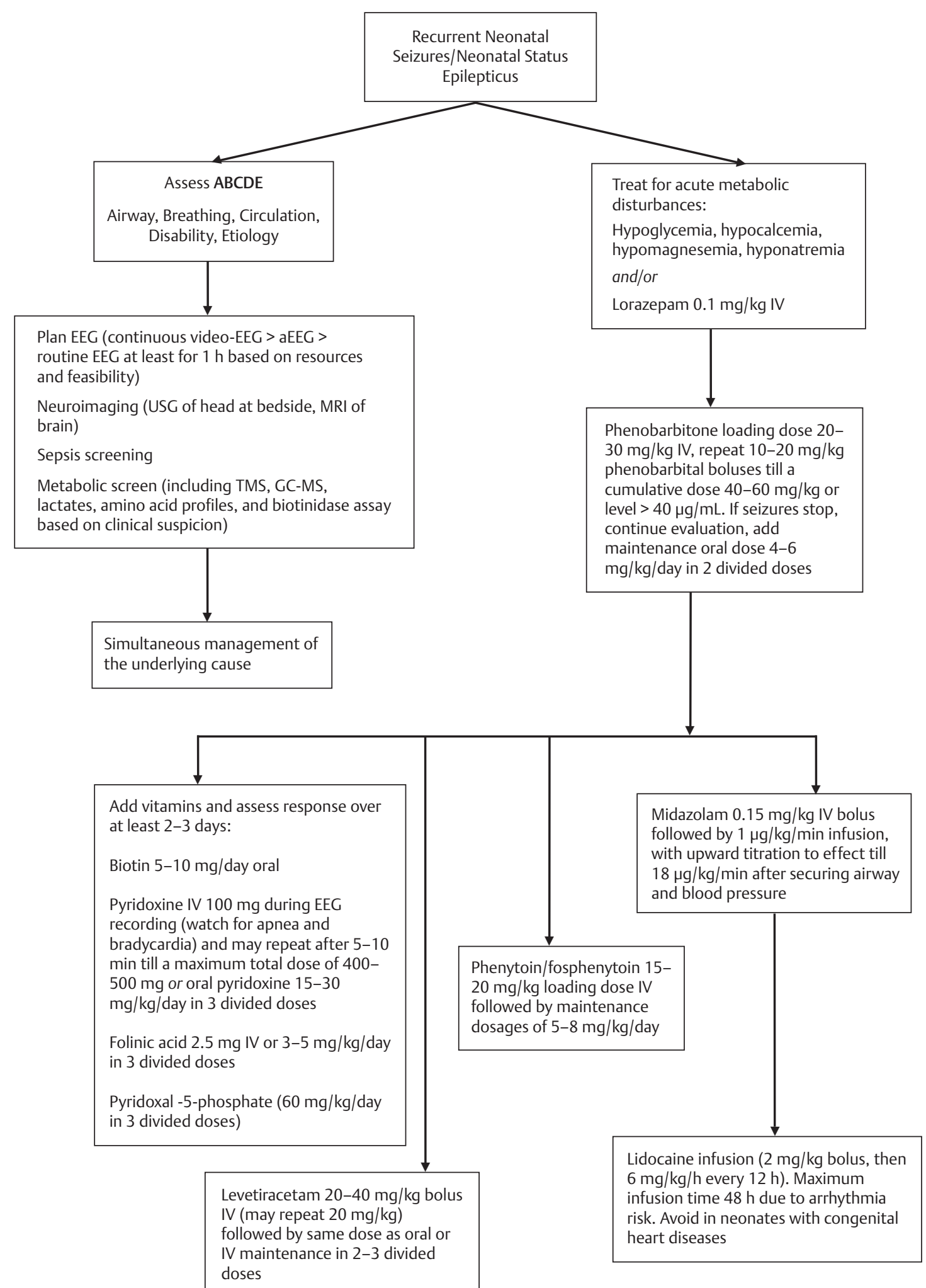

Fig. 1 Algorithmic approach to recurrent neonatal seizures. aEEG, amplitude-integrated EEG, EEG, electroencephalography; GC-MS, gas chromatography-mass spectrometry; MRI, magnetic resonance imaging; TMS, tandem mass spectrometry; USG, ultrasonography.

had to be stopped prematurely. ${ }^{47}$ This highlights the risks associated with the off-label use of drugs in newborn infants before safety assessment in controlled trials. Standardized, protocol-driven treatments improve consistency and shortterm outcome in NSE and should be practiced at all centers keeping in mind the resource constraints. ${ }^{48}$ 


\section{Outcomes}

Although the immature brain is proposed to be more resistant to seizures and status-induced injury than an adult brain, several neurobiological changes such as disruption of neuronal development and networks, induction of synaptic reorganization, altered plasticity, molecular reorganization of receptors, and priming of the brain for secondary and tertiary injury has been reported. ${ }^{49}$ Status epilepticus has been shown to be an independent predictor of adverse neurological outcome and epilepsy at 2 years' corrected age. ${ }^{2}$ The risk of development of epilepsy after early-life status epilepticus has been estimated at 13 to $74 \% .^{50}$ A relationship has been proposed between NSE and classic unilateral hippocampal sclerosis and temporal lobe epilepsy. ${ }^{51}$ The presence of status epilepticus significantly increases the odds for development of cerebral palsy, global developmental delay, and epilepsy. ${ }^{52}$ The effect of gestational age has not been significant on the outcomes. ${ }^{20,48}$ Febrile status epilepticus has been more frequently reported in children with a history of neonatal seizures and neurological abnormality. ${ }^{53}$

\section{Conclusion}

In conclusion, NSE is marker of neonatal brain injury and needs urgent evaluation, and the broad principles of management are extrapolated from guidelines for children and adults. As majority of NSEs are provoked, simultaneous treatment of the underlying etiology constitutes an important aspect of management. Further research is needed into the role of current antiepileptic drugs in causing neuronal injury and use of neuroprotective agents during NSE.

\section{Funding}

None.

\section{Conflict of Interest}

None.

\section{References}

1 Volpe J. Neonatal seizures. In: Neurology of the Newborn. Philadelphia, PA: W.B. Saunders; 2001:178-214

2 Pisani F, Cerminara C, Fusco C, Sisti L. Neonatal status epilepticus vs recurrent neonatal seizures: clinical findings and outcome. Neurology 2007;69(23):2177-2185

3 Jonas P, Racca C, Sakmann B, Seeburg PH, Monyer H. Differences in $\mathrm{Ca} 2+$ permeability of AMPA-type glutamate receptor channels in neocortical neurons caused by differential GluR-B subunit expression. Neuron 1994;12(6):1281-1289

4 Zhong J, Carrozza DP, Williams K, Pritchett DB, Molinoff PB. Expression of mRNAs encoding subunits of the NMDA receptor in developing rat brain. J Neurochem 1995;64(2):531-539

5 Dunning DD, Hoover CL, Soltesz I, Smith MA, O'Dowd DK. GABA(A) receptor-mediated miniature postsynaptic currents and alpha-subunit expression in developing cortical neurons. J Neurophysiol 1999;82(6):3286-3297

6 Khazipov R, Khalilov I, Tyzio R, Morozova E, Ben-Ari Y, Holmes GL. Developmental changes in GABAergic actions and seizure susceptibility in the rat hippocampus. Eur J Neurosci 2004;19(3):590-600
7 Dzhala VI, Talos DM, Sdrulla DA, et al. NKCC1 transporter facilitates seizures in the developing brain. Nat Med 2005;11(11):1205-1213

8 Mikati MA, Werner S, Shalak L, et al. Stages of status epilepticus in the developing brain. Epilepsy Res 2003;55(1-2):9-19

9 Berg AT, Shinnar S, Levy SR, Testa FM. Status epilepticus in children with newly diagnosed epilepsy. Ann Neurol 1999;45(5):618-623

10 Lowenstein DH. Status epilepticus: an overview of the clinical problem. Epilepsia 1999;40(Suppl 1):S3-S8, discussion S21-S22

11 Trinka E, Cock H, Hesdorffer D, et al. A definition and classification of status epilepticus-report of the ILAE Task Force on Classification of Status Epilepticus. Epilepsia 2015;56(10):1515-1523

12 Smith DM, McGinnis EL, Walleigh DJ, Abend NS. Management of status epilepticus in children. J Clin Med 2016;5(4):5

13 Berg AT, Berkovic SF, Brodie MJ, et al. Revised terminology and concepts for organization of seizures and epilepsies: report of the ILAE Commission on Classification and Terminology, 2005-2009. Epilepsia 2010;51(4):676-685

14 Mizrahi EM. Acute and chronic effects of seizures in the developing brain: lessons from clinical experience. Epilepsia 1999;40(Suppl 1):S42-S50, discussion S64-S66

15 Prasad AN, Seshia SS. Status epilepticus in pediatric practice: neonate to adolescent. Adv Neurol 2006;97:229-243

16 Wusthoff CJ, Dlugos DJ, Gutierrez-Colina A, et al. Electrographic seizures during therapeutic hypothermia for neonatal hypoxic-ischemic encephalopathy. J Child Neurol 2011;26(6):724-728

17 Pisani F, Orsini M, Braibanti S, Copioli C, Sisti L, Turco EC. Development of epilepsy in newborns with moderate hypoxic-ischemic encephalopathy and neonatal seizures. Brain Dev 2009;31(1):64-68

18 Wertheim D, Mercuri E, Faundez JC, Rutherford M, Acolet D, Dubowitz L. Prognostic value of continuous electroencephalographic recording in full term infants with hypoxic ischaemic encephalopathy. Arch Dis Child 1994;71(2):F97-F102

19 McBride MC, Laroia N, Guillet R. Electrographic seizures in neonates correlate with poor neurodevelopmental outcome. Neurology 2000;55(4):506-513

20 Pavlidis E, Spagnoli C, Pelosi A, Mazzotta S, Pisani F. Neonatal status epilepticus: differences between preterm and term newborns. Eur J Paediatr Neurol 2015;19(3):314-319

21 Lawrence R, Inder T. Neonatal status epilepticus. Semin Pediatr Neurol 2010;17(3):163-168

22 Shellhaas RA, Clancy RR. Characterization of neonatal seizures by conventional EEG and single-channel EEG. Clin Neurophysiol 2007;118(10):2156-2161

23 Lawrence R, Mathur A, Nguyen The Tich S, Zempel J, Inder T. A pilot study of continuous limited-channel aEEG in term infants with encephalopathy. J Pediatr 2009;154(6): 835-41.e1

24 Silverstein FS, Jensen FE. Neonatal seizures. Ann Neurol 2007;62(2):112-120

25 Wusthoff CJ. Diagnosing neonatal seizures and status epilepticus. J Clin Neurophysiol 2013;30(2):115-121

26 Lombroso CT. Neonatal seizures: gaps between the laboratory and the clinic. Epilepsia 2007;48(Suppl 2):83-106

27 Vasudevan C, Levene M. Epidemiology and aetiology of neonatal seizures. Semin Fetal Neonatal Med 2013;18(4):185-191

28 Vinayan KP, Moshe SL. Neonatal seizures and epilepsies. Int J Epilep 2014;1:75-83

29 Dlugos DJ. The nature of neonatal status epilepticus-a clinician's perspective. Epilepsy Behav 2015;49:88-89

30 Hart AR, Pilling EL, Alix JJ. Neonatal seizures-part 1: not everything that jerks, stiffens and shakes is a fit. Arch Dis Child Educ Pract Ed 2015;100(4):170-175 
31 Saini AG, Taketani T, Sahu JK, Singhi P. Startles, stiffness, and SLC6A5: do you know the condition? Pediatr Neurol 2018;81:49-50

32 Abend NS, Wusthoff CJ, Goldberg EM, Dlugos DJ. Electrographic seizures and status epilepticus in critically ill children and neonates with encephalopathy. Lancet Neurol 2013;12(12):1170-1179

33 Silverstein FS, Jensen FE, Inder T, Hellstrom-Westas L, Hirtz D, Ferriero DM. Improving the treatment of neonatal seizures: National Institute of Neurological Disorders and Stroke workshop report. J Pediatr 2008;153(1):12-15

34 Clancy RR. Summary proceedings from the neurology group on neonatal seizures. Pediatrics 2006;117(3 Pt 2):S23-S27

35 Shellhaas RA, Chang T, Tsuchida T, et al. The American Clinical Neurophysiology Society's Guideline on Continuous Electroencephalography Monitoring in Neonates. J Clin Neurophysiol 2011;28(6):611-617

36 Sankar R, Shin DH, Liu H, Mazarati A, Pereira de Vasconcelos A, Wasterlain CG. Patterns of status epilepticus-induced neuronal injury during development and long-term consequences. J Neurosci 1998;18(20):8382-8393

37 Shah DK, Wusthoff CJ, Clarke P, et al. Electrographic seizures are associated with brain injury in newborns undergoing therapeutic hypothermia. Arch Dis Child Fetal Neonatal Ed 2014;99(3):F219-F224

38 Painter MJ, Scher MS, Stein AD, et al. Phenobarbital compared with phenytoin for the treatment of neonatal seizures. N Engl J Med 1999;341(7):485-489

39 Hellström-Westas L, Boylan G, Ågren J. Systematic review of neonatal seizure management strategies provides guidance on anti-epileptic treatment. Acta Paediatr 2015;104(2):123-129

40 Glass HC, Kan J, Bonifacio SL, Ferriero DM. Neonatal seizures: treatment practices among term and preterm infants. Pediatr Neurol 2012;46(2):111-115

41 Gospe SM Jr. Neonatal vitamin-responsive epileptic encephalopathies. Chang Gung Med J 2010;33(1):1-12

42 Krief P, Li Kan, Maytal J. Efficacy of levetiracetam in children with epilepsy younger than 2 years of age. J Child Neurol 2008;23(5):582-584
43 Khan O, Chang E, Cipriani C, Wright C, Crisp E, Kirmani B. Use of intravenous levetiracetam for management of acute seizures in neonates. Pediatr Neurol 2011;44(4):265-269

44 Talos DM, Chang M, Kosaras B, et al. Antiepileptic effects of levetiracetam in a rodent neonatal seizure model. Pediatr Res 2013;73(1):24-30

45 Jensen FE. Neonatal seizures: an update on mechanisms and management. Clin Perinatol 2009;36(4):881-900, vii

46 Srinivasakumar P, Zempel J, Wallendorf M, Lawrence R, Inder $\mathrm{T}$, Mathur A. Therapeutic hypothermia in neonatal hypoxic ischemic encephalopathy: electrographic seizures and magnetic resonance imaging evidence of injury. J Pediatr 2013;163(2):465-470

47 Pressler RM, Boylan GB, Marlow N, et al; NEonatal seizure treatment with Medication Off-patent (NEMO) consortium. Bumetanide for the treatment of seizures in newborn babies with hypoxic ischaemic encephalopathy (NEMO): an open-label, dose finding, and feasibility phase $1 / 2$ trial. Lancet Neurol 2015;14(5):469-477

48 Harris ML, Malloy KM, Lawson SN, Rose RS, Buss WF, Mietzsch U. Standardized treatment of neonatal status epilepticus improves outcome. J Child Neurol 2016;31(14):1546-1554

49 Akman O, Moshé SL, Galanopoulou AS. Early life status epilepticus and stress have distinct and sex-specific effects on learning, subsequent seizure outcomes, including anticonvulsant response to phenobarbital. CNS Neurosci Ther 2015;21(2):181-192

50 Raspall-Chaure M, Chin RF, Neville BG, Scott RC. Outcome of paediatric convulsive status epilepticus: a systematic review. Lancet Neurol 2006;5(9):769-779

51 Dunleavy M, Shinoda S, Schindler C, et al. Experimental neonatal status epilepticus and the development of temporal lobe epilepsy with unilateral hippocampal sclerosis. Am J Pathol 2010;176(1):330-342

52 Yıldız EP, Tatlı B, Ekici B, et al. Evaluation of etiologic and prognostic factors in neonatal convulsions. Pediatr Neurol 2012;47(3):186-192

53 Shinnar S, Pellock JM, Berg AT, et al. Short-term outcomes of children with febrile status epilepticus. Epilepsia 2001;42(1):47-53 